\title{
\%A BEOWULF.
}

Es fehlt leider eine gut commentierte ausgabe des Beowulf, welche jede irgendwie bedenkliche stelle historisch beleuchtete. Grade die alteren editoren haben manchen guten gedanken gehabt, der nie recht zur geltung gekommen ist. Vielleicht werden die angekündigten neueren ausgaben gegen Heyne einen scheinbaren rückschritt tun mïssen um oft besseres zu bieten. Eine verwertung und kritik aller bisherigen ansichten würde die schwierigen stellen mehr in den vordergrund drängen, unnötige reflexionen über längst erledigte probleme blieben erspart und man liefe nicht leicht gefahr gesagtes zu widerholen. So haben denn die neueren aufsätze von Cosijn und Sievers (Beitr. VIII, 568; IX, 136) nicht an Heynes Beowulfausgabe angeknüpft, weil sie keineswegs die früheren erörterungen zusammenfasst und abschliesst. Aber besonders Sievers anmerkungen zeigen, dass es schon nicht mehr leicht ist die reiche literatur ganz zu überschauen. Sie enthalten beobachtungen, die schon vor ibm gemacht wurden ohne des letzten herausgebers beifall $\mathrm{zu}$ finden. roden fur hroden 1151 hatte Bugge 'Tidskr. VIII, 64. 295 rorgeschlagen und Rieger Zachers zs. III, 404 sowie Gering ebenda XII, 151 f. empfoblen. 1546 batte Ettmüller Scop.-Bôc. s. 119 (nach ihm Sweet Ags. Reader s. 129) stillschweigend seaxe in seax geändert. Die von Sievers vertretene auffassung von 1557 steht schon in Ettmullers text ebeuda; erst Mullenboff Haupts zs. XIV, 210 scheint die in unsern neueren ausgaben geltende auffassung angeregt zu haben. Heynes verfehlte interpunktion v. 2594 hatte Ettmaller s. 123 vermieden. Sievers' vorschlag zu 3097 erneuert Bugges bemerkung 'lidskr. VIII, 300, wie aus Heynes note z. d. st. hervorgeht. Leider sind mir die engl. Beowulfausgaben nicht zugïnglich, und so bin ich nicht sicher, ob nicht auch unter den folgenden bemerkungen einige sind, welche bereits vorgebracht sind; das deutsche material glaule ich jedenfalls nicht zu widerholen. Ich darf mir schliesslich die bemerkung gestatten, dass bei einer eingehenden Beowulflektüre, welche ich vor dem erscheinen von Cosijns aúfsatz mit zwei freunden begann, cinige coniecturen in unserm kreise aufkamen, die wir bald 
darauf von Cosijn und Sievers vertreten fanden; dies gilt von Cosijns bemerkungen zu v. 120. 901 und von Sievers' annahme von strĭde für strâde v. 3075 .

31. Man erwartet für lange etwa landagas; oder sollte leof leodfruma land âhte $\mathrm{zu}$ ändern sein?

112. Bugges erklärung von orcneas kann ich nicht billigen: eine ableitung aus orcus mit dem eas von Persêas, Judêas ergibt noch immer kein orcneas', auch nicht die bedeutung 'seeungeheuer'. Auch Greins zuziehung von $n \hat{e}$ 'cadaver' gentigt nicht. Mir scheint Heyne früher auf dem richtigen wege gewesen $z u$ sein, als er altnorweg. orkn 'phoca barbata' zuzog. Bugges zweifel dagegen werden unberechtigt, wenn man orcneas als compositum gleich orcen-eoh (eas für eos plur. verschrieben) nimmt: dann genügt der vers metrisch.

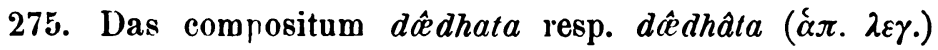
gibt keinen sinn. Man ändere in das häufige dî́dhwata.

360. Fasst man feorrancumen als substantiviertes compositum 'fremdling' (so erscheint das wort in der prosa), so erhält die stelle einen schlichteren stil; Geata leode ist dann apposition resp. nähere detaillierung zu feorrancumene.

444. unforhte klingt absurd; es ist entweder anforhte 'timidos' oder praedicativisch unforht 'impavidus' zu lesen.

490 fasse ich onsếl meoto als 'entseile die etikette, löse die fesseln der etikette'; ein compositum sigehrểsecg 'ruhmesheld' wäre unerhört; ich vermute sigehrêðegum (-hrêðgum) für -hrềsecyum.

586. Der ausfall einer langzeile ist sehr fraglich, da der ausfall eines wortes wie geflites ('wettschwimmen') den sinn lierstellt. 'Weder Breca noch du habt jemals solchen kampf bestanden - ohne mich jenes wettschwimmens ruhmen zu wollen -, obwol du doch allbekanntes, berichtigtes hast vollbringen können.' Greins ergänzung von fela lässt unklarheit uber die beziehung.

650. Die periode ist von Heyne unschön gebaut. Für werod eall ârâs vermisst man eine zeitbestimmung, wie sie in der nachahnung Andr. 835 steht. Offenbar erfullt 651 und 652a diesen zweck; es ist somit nach 650 ein punkt zu setzen. 
695. hie ar: es fehlt der genitiv zu fela, man indere hiera 'zu viel von ihnen', parallel Denia leode 'vom volke der Dänen' (Heyne s. v. fasst leode richtig als genet.).

856 fasse ich ridan nicht als inf. praes. (ridan), souderu als praet. plur. (ridon).

992. Bugges änderung heahtimbred für hâten hreðe ist etwas complicicrt; einfacher ist es fir hâten etwa hroden zu schreiben, so dass folmum fretenod variation wird.

1032. Wäre nicht wala ( $\breve{a}$ ? $\hat{a}$ ?) 'helmbusch, rossschweif' möglich mit rlicksicht auf das gleichbed. skr. vâra? Vgl. auch ahd. wâla 'flabellum'.

1075. gebyrd als 'fatum' ist kaum zweifelhaft; vgl. ahd. giburt 'sors' Graff III, 160.

1232. Eine änderung von dồ in dô liesse sich empfehlen, wenn dadurch das ganze in ordnung käme. Auch lïsst sich gegen die bisherige auffassung soviel ich sehe nichts vorfuhren; zu ihren gunsten spricht der umstand, dass sich v. $1233 \mathrm{ff}$. uber die sämmtlichen teilnehmer des gelages auslassen und nicht speciell mit Beowulf anknlipfen. Der fehler scheint mir etwas fruher zu stecken. Das wort dreamhealdende halte ich für eine anrede der königin an die ganze versammlung; dream kann sich eben nicht auf den jubel des einzelnen beziehen ('lætabundus'), sondern nur auf das fröbliche treiben des gelages. Dann ergibt sich als naheliegend die änderung von is in $s i(s \hat{\imath})$; und wenn dem schreiber einmal is für sî in den sinn und in die feder $\mathrm{kam}$, so musste auch ein sin einem sindon (resp. sind) platz machen. Darnach wäre der schluss von Wealhpeows rede an das ganze gelage gerichtet, und die worte enthielten eine aufforderung an die eorlas zu treuer friedlicber gesinnung, an die pegnas $\mathrm{zu}$ bereitwilligem gehorsam. dôp $s w \hat{a}$ ic bidde schliessen diese ermahnungen eindringlich ab.

1254. Für warode lesen Kölbing, Holder, Wülcker farode; die stelle bedarf zweifelsohne eines anderen wortes als warode v. 1266. Ettmüller vermutete fêrode; ich halte im anschluss daran nach dem im Anz. d. Angl. behandelten gesetz an fârôde durchaus fest, vgl. as. fârôn 'auflauern'.

1402. geatolic: Dass die stets angenommene bedeutung 'stattlich' den sirn nicht trifft, ergiebt die etymologie des wortes: es steht für gatul-lîk (cf. atelîc für atollik im Beow.); 
westgerm. gri-talo- crscheint im ahd. nit gewechselter betonung als gi-zal, 'alacer, agilis, flink, gewant' und wird besonders von rossen und pfeilen gebraucht; gizeliro 'melior pedibus'. 'Leicht sich bewegend' scheint die berleutung des wortes zu sein; so hicr. Vom speer gebraucht erscheint das wort 215 (searo 'speer' hat Bugge nachgewiesen), ähnlich vom schwert 1563. 2155. Auf weiterer entwicklung, bei der einwirkung von geatwe möglich, nimmt das wort die bedeutung 'praechtig' an. Es ist eines von den altertumlichen worten des Beow., in seiner jüngsten bedeutung vom dichter der Elene erneuert.

1862 dürfte headu in heafu (pl. zu hœf) zu ändern sein. Jedenfalls steht die herrschende erklärung aus heahðu im widerstreit mit der suffixlehre und den lautregeln. Auch die stets daraus erklärte heaðolı̂̉ende 1799. 2956 beweist kein headu 'mer'. Warum kann diese zusammensetzung, welche ein nachahmer des Beowulfepos in den Andreas brachte, nicht 'kampfsecfahrer' bedeuten? heádu ist undenkbar, weil dafür hei $i$ eintreten müsste; daher ist heaðu sicher. Also ist auch heaðosigl 'sol e mari progrediens' Räts. 72 bedenklich.

1876 f. will Sievers eine reihe änderungen anbringen, die mir mit riicksicht auf die deutliche nachahmung unserer stelle Andr. 1012 unnötig erscheinen. Diese dichtung, welche voll von reminiscenzen an den Beow. ist, kann auch sonst zur beleuchtung textkritischer und interpretationsfragen des Beow. benutzt werden, wie denn Sievers' anmerkung zu 1858 durch Andr. 1013 gestlutzt wird. Nun vergleiche man Andr. paes- $\partial e$ hie onsunde $\hat{c}$ fre môslon geseon under sunnan mit Beow. pat hie (für he) seoðठ̇an geseon môston môdige on meðle: also geseon 'sich wieder sehen'.

$2031 \mathrm{ff}$. Ich beginne mit lytle hwîle einen neuen satz, weil oft in diesen satz nicht passt, da der abhängige satz peah seo brŷtl duge trotz Lichtenheld Haupts zs. 16,343 eine sentenz ausschliesst. Hrồgâr hegt die absicht, durch die verlobung der Frcawaru die feindseligkeiten gegen die Barden beizulegen, nachdem er leute genug im kampfe verloren ( mit oft seldan hwcer weiss ich ebensowenig etwas anzufangen als die bisherigen erklärer). Doch nur kurze zeit werden die waffen ruhen, so zufriedenstellend auch der kontrakt und die damit verbundene verlobung ist (f)eah seo brŷd duge). Im 
verlauf ler prophezeiungen Beowulfs scheint noch nicht bemerkt zu sein, dass nicht bloss der sinn, sondern auch der wortlaut verlangt, unter den dryhtbearn (dryhtbeorn?) Dena den brautfiihrer der Freawiru zu vorstehen: dryhtealdor 'paranymphus' und grleichbed. dryhtguma sind aus gloss. bezeugt und stehen in uraltem zusammenhang mit ahd. truhtgomo, truhting, as. druhting, longobard. droctinc 'brautführer'. Durch se fếmnan pegn 2060 wird unsere auffassung von dryhtbeorn nötig. 2033 scheint peodne nöti.r.

2196. 2995 ergïnzt man neuerdings nach Riegers crörterung Zachers zs. III, 415 zu pûsendo cine münze als masscinheit; aber scealt ist nie als allgemeingültige werteinheit sebraucht; jedenfalis war der scẹatt, obzwar in verschiedenen gegenden von verschiedenem wert, die allergeringste mïnze, und es wundert mich wic Rieger bei seiner auffassung unserer stellen auf Beow. 1686 sceqattas dcelde verweisen konnte, wo das wort nur geld und geldesivert im allgemeinen bedeutet. Aus der reichbaltigen erörterung von Schmid Ges. ${ }^{2} 591 \mathrm{f}$. geht deutlichst hervor, dass keine allgemeine geldeinheit bestand, ron der hier die rede sein könnte. Auch kennen wir aus der ac. poesie keine solche dedicationen. Was unter seofon phsendo in verbindung mit bold and bregostôl $2197 \mathrm{zu}$ versteben ist, kann 2493 he mê land forgẹf, eard ề elwynn zcigen (v. 2497 weorde chypan mag immerhin auf geldeswert als lohn für die recken deuten). Instructiv ist auch Andr. $301-3103$; ncebbe ic fâtedgold ne jeohgestreon, welan ne wiste ne wira gespann, landes ne locenra beaga antwortet Andreas dem herren der als schiffer ihn ubersetzen will, wofern er die gebihr entrichte

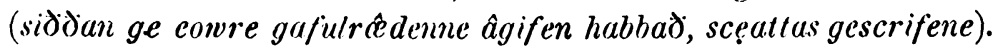
Kurz ich glaube mit Ettmiller, dass zu pisendo land resp. ein bestimmte masseinheit von land $z u$ verstehen ist. Dass diese nasseinheit nur ein hîde gewesen sein kann was Ettmiiller übersah, ergibt sich mit voller sicherheit aus dem reichen material, das Schmid in den angls. gesetzen $: 610 \mathrm{zu}-$ sammengetragen hat. Mich hat die lecture von Bedas kirchenreschichte zu dieser auffassung geführt: hier kommen die landschenkungen so zahlreich vor und zwar jedesmal mit der genannten masseinheit, die er auch in seinen geographischen angaben immer verwendet (I, 25. II, 9. III, 4. 24. IIII, 13. 
16. 19. V, 19). Besonders erinnere ich an III, 34, wo es liber den könig Oswiu heisst: donavit praefato Peada, filio regis Pendan, co quod esset cognatus suus, regnium Australium Merciorum, qui sunt familiarum quinque milium. Auch die in Beow. 2196 und 2995 berichteten schenkungen geschehen an verwante (2432 sibbe gemunde $=$ eo quod cognatus esset). Es mag noch erwähnt werden, dass Bedas ubbersetzung von hìd durch familia der ausgangspunkt der etymologie des wortes sein muss (ef. Bosworth-Toller s. hîd). Vgl. noch die folgende anmerkung. Die bestimmung der hîde ergibt sich sehr bequem nach Beda, der die grösse der insel Wight auf 1200 hîden, die der insel Thanet auf 600 biden bestimmt. Nun ist die insel Wight $400 \square$ kilom. gross; also 1 hîde $=1 / 3 \square$ kilom. Das dem Beowulf geschenkte territorium von 7000 hiden nicht viel mehr als Oswiu's geschenk an seinen verwandten Peada - umfasst etwa $2300 \square$ kilom., also etwa $40 \square$ meilen. Naturlich ist seofon ebenso wie hund 2995 nur des reimes wegen gewählt, und man darf somit die eben gemachte berechnung nicht als ernsthaft nehmen.

2607. Die herrschende auffassung von âre als 'gnade, huld' ist mir zweifelhaft. Man scheint die in urkunden so häufige verwendung ron $\hat{a} r$ 'besitz', besonders 'liegenschaft' (land-ar) nicht fur die pocsie anzuerkennen. Dies ist naturlich ganz verschieden von $\hat{a} r$ 'huld, schonung, gnade', wie bereits Schmid im glossar zu den gesetzen s. $\hat{a} r$ richtig erkannte; $\hat{a} r$ 'grundbesitz' gehört zu ah, got. aih 'habe'. are ist plural.

2706. ferh ellen wrac als parenthese ist auffällig, mag man mit Grein ellen oder mit Heyne ferh als subjekt nehmen. Ich schlage feorh ealne wrac vor; feorh wrecan 'das leben austreiben' belegt Grein noch aus Genes. 1385. So erhält durch diese wie mir scheint nötige auffassung die von Sievers befürwortete konjektur 'Thorpe's gefylde für gefyldan eine weitere stuize.

2767. Greins auffassung von oferhigian 'supereminere' ist so haltlos wie Bugges erklärung des wortes aus einem got. ubarhauhjan (-jôn?). Heynes berufung auf ahd. ubarlucken 'ubermbitig sein' und seine annahme der bedeutung 'ubermuitig machen, betören' fthlıren auf die vermutung: oferhŷdgian zu oferhŷd, oferhygd. 